\title{
Truth Graph: A Novel Method for Minimizing Boolean Algebra Expressions by Using Graphs
}

\author{
Eisa Alharbi $\left.{ }^{(}\right)$ \\ Kuwait Oil Company, Ahmadi, Kuwait \\ ealharbi@kockw.com
}

\begin{abstract}
Boolean algebra expressions are used by stakeholders from a range of disciplines, such as engineers, to build logic circuits. To reduce the cost and transaction numbers of logic circuits, engineers minimize Boolean expressions by reducing the number of terms or arithmetic operations. A well-known pictorial method applied for minimizing Boolean expressions is the Karnaugh map. However, a drawback is that these maps are not effective for minimizing Boolean expressions involving more than four variables. We introduce a novel method for minimizing Boolean expressions by using graphs, which we call a "Truth Graph" that can be effectively applied when many variables exist.
\end{abstract}

Keywords: Boolean algebra $\cdot$ Minimizing Boolean expressions

\section{Introduction}

Stakeholders, such as engineers, use Boolean algebra expressions for building logic circuits. To reduce the cost of these circuits, they must reduce (or minimize) the original Boolean expressions to an equivalent expression that includes fewer terms or arithmetic operations. The process of minimizing Boolean expressions is not straightforward, and understanding circuits and the connections between its components is challenging for engineering students [13]. Geoffrey et al., who studied students' misconceptions of circuits, revealed that " these misconceptions result from the need to manage a lot of information that has not been properly organized in the students' minds." [9].

Tools, such as truth tables and the Karnaugh map (K-map), were introduced to help simplify minimizing Boolean expressions [14]. However, these tools have drawbacks. Truth tables grow very fast as the number of rows for an n-variable function is $2^{n}$. Therefore, having more than five variables, for example, will make the construction of the table laborious and prone to error.

The K-map is another form of the truth table that facilitates the minimization of Boolean algebra expressions without requiring the use of Boolean algebra theorems. However, the K-map becomes highly confusing when minimizing expressions that involve more than four variables. For example, a 4 -variable KMap will include $2^{4}=16$ cells, each having a value of either 0 or 1 . These 16 cells consist of four rows and four columns, each labelled with two binary numbers 
that bring the total binary number in a 4-variable K-Map to 32 . Analyzing the relations between these binary numbers by grouping adjacent cells that contain the one values to determine a minimum expression can also be arduous and prone to error. Studies demonstrated that students find K-maps difficult to use, such as Zilles et al. who interviewed students taking a logic course that incorporated using K-maps, and reported,"because Karnaugh maps are a major topic in both ECE 290 and CS 231, we were surprised to find that students were generally reluctant to use them." [15]. Considering that both truth tables and the K-map are utilized as pedagogical tools, having an enhanced teaching approach that supports many variables and reduced visual complexity would be valuable.

Learning with an appropriate representation can enhance learners' performance [1]. Cromley investigated if different representations led learners to use different strategies and reported that "students verbalize more inferences when reading diagrams compared to text" [8]. The superiority of visual notations over textual and symbolic versions has been reported by Larkin and Simon [10], Cheng [7], and many others, including Ainsworth and Loizou [2], Alharbi [3], and Butcher [5].

By considering the superiority of visual notations, especially in logic with their support for observational advantages and reasoning [11,12], in terms of visual complexity and drawability $[4,6]$, visual notations can be effective representations for minimizing Boolean expressions. This paper introduces a new method for minimizing Boolean algebra expressions that takes advantage of the benefits of visual representations to overcome the limitations of truth tables and the K-Map approaches.

\section{Truth Graph Expressions}

A truth table is a simple tool for showing the truth-value of all possible combinations of the variables within an expression. Combinations leading to false outputs are not involved in the minimization process. Therefore, truth graphs only represent combinations that lead to truth outputs. Figure 1 and Table 1 illustrate how a truth graph and truth table represent the Boolean expression $(A+B) * C$ with values. In Fig. 1, each variable can have one of two values, 1 or

Table 1. Truth values.

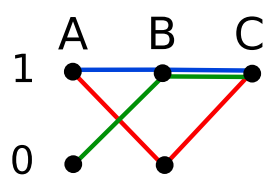

Fig. 1. Truth graph. (Color figure online)

\begin{tabular}{|c|c|c|c|}
\hline $\mathrm{A}$ & $\mathrm{B}$ & $\mathrm{C}$ & Output \\
\hline 0 & 0 & 0 & 0 \\
\hline 0 & 0 & 1 & 0 \\
\hline 0 & 1 & 0 & 0 \\
\hline 0 & 1 & 1 & 1 \\
\hline 1 & 0 & 0 & 0 \\
\hline 1 & 0 & 1 & 1 \\
\hline 1 & 1 & 0 & 0 \\
\hline 1 & 1 & 1 & 1 \\
\hline
\end{tabular}


0 . The upper nodes represent the 1 values, and lower nodes represent 0 . Values not included in the graph do not lead to truth outputs, such as the value 0 for variable $\mathrm{C}$ is not included in the graph.

Values (or nodes) are connected by edges that represent truth paths. In Fig. 1, there exist three truth paths highlighted in green, red, and blue. These truth paths represent the truth outputs of the combinations of the values. For example, in the green path, the value 0 from $\mathrm{A}$ is linked with the value 1 of $\mathrm{B}$, which is linked with the value 1 of $\mathrm{C}$. This truth path is read as 011 or FTT, which is equivalent to the fourth row in Table 1 . The red path represents the values $1 \mathrm{~A}, 0 \mathrm{~B}$, and $1 \mathrm{C}$, and the blue path represents the values $1 \mathrm{~A}, 1 \mathrm{~B}$, and $1 \mathrm{C}$. If there exists no edge (link) between two values, then the combination of these values is false. For example, no link exists between $0 \mathrm{~A}, 0 \mathrm{~B}$, and $0 \mathrm{C}$.

In the graph, we include nodes with written values of 1 and 0 to illustrate the idea. These elements can be eliminated to make the graph simpler without impacting the semantics. Figure 2 shows the same graph after removing the nodes and written values, which reduces the visual complexity and transforms the graph into a representation similar to electrical signals that can be read easily and matched to its meaning, where high waves represent 1 values and low waves represent 0 . This version of the graph appears more straightforward and easier to draw compared to a truth table, especially with many variables.

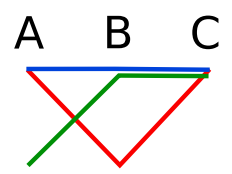

Fig. 2. Truth graph after removing nodes and written values.

\section{Truth Graph Minimization Method}

Reducing the number of variables or connections corresponds to minimizing Boolean expressions. The truth graph method minimizes Boolean expressions by following two rules:

If two paths pass through the same variables and values, except for one variable where one path passes through 1 and the other passes through 0 , then this variable can be eliminated from the two paths. For example, $A B C+\bar{A} B C=B C$.

If one path passes through fewer variables than another path, and the shortest path passes through the same variables and values, except for one variable, then the value can be eliminated only from the path that has more variables. For example, $A B+\bar{A} B C=A B+B C$.

Regardless of the values, in the first rule, two expressions must have the same variable, and in the second rule, one expression is subsumed by another. In Fig. 2, the green and blue paths pass through the same values, except the values of variable A. Therefore, these two paths can be minimized to BC. In Fig. 3, the 
black path represents the combined result of the green and blue paths. Next, we combine this result with the red path. Both black and red paths pass through the values 1 and 0 of variable $B$, and the red path passes by more values. Therefore, we eliminate the value of variable $B$ from the red path. The result of the red path becomes AC.

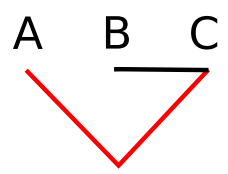

Fig. 3. The result of minimizing the green and blue paths. (Color figure online)

The final minimization result of the truth graph in Fig. 2 becomes $\mathrm{AC}+\mathrm{BC}$, as shown in Fig. 4. The dashed line is used instead of a solid line to avoid visual complexity and illustrates that A is linked to $\mathrm{C}$ without $\mathrm{B}$. The solid line links all variables it passes through, where the dashed line skips some variables. In this example, the dashed line passes through $\mathrm{AB}$ and $\mathrm{C}$, and the small black dots below the variables $\mathrm{A}$ and $\mathrm{C}$ represent that $\mathrm{A}$ is linked to $\mathrm{C}$ without $\mathrm{B}$.

\section{A $\mathrm{B} \quad \mathrm{C}$}

Fig. 4. The result of minimizing the three paths. (Color figure online)

The final minimization result is not affected by the order of the combinations because combining any two paths first will lead to the same result. For example, we can start by combining the red and blue paths by eliminating the variable $\mathrm{B}$. The result is AC, as shown in Fig. 5. The black dotted line represents the result of minimizing the red and blue paths. Then, the black dotted and green paths can be minimized by eliminating the value 0 of variable $\mathrm{A}$ from the green path. The reason for eliminating the value of A from the green path only is because the green path passes through more variables than the black path, which passes through only two variables. The result is the same as the previous result, as shown in Fig. 4.

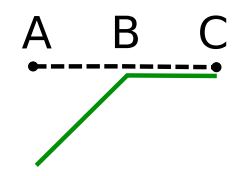

Fig. 5. The result of minimizing the green and blue paths. (Color figure online) 
The minimization rule cannot be applied to the combination of the green and red paths because the two paths pass through three values and share only one value. Figure 6 shows several examples of combining truth paths that cannot be minimized. The truth paths in graphs a and b do not share any values, and the truth paths in graphs $\mathrm{c}, \mathrm{d}$, e, and $\mathrm{f}$ share only one value as they pass through three values. The truth paths in graphs $g$ and $h$ share two values as they pass through four values.

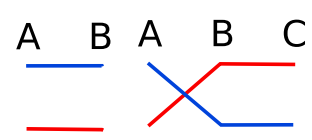

(a) (b)

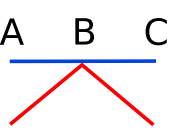

(c)

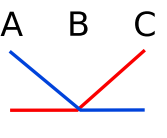

(d)

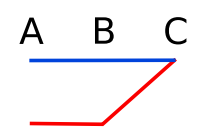

(e)

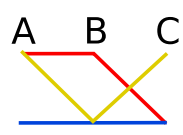

(f)

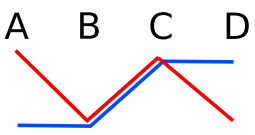

(g)

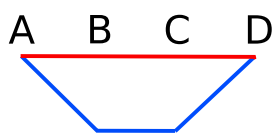

(h)

Fig. 6. Examples of graphs that cannot be minimize. (Color figure online)

\section{Truth graph and K-Map (Three Variables)}

K-map cells filled with either 1 s or 0 s, where the 1 values indicate that the true values of the combination and 0 values indicate the falsity. To find the minimum logic function, these 1 values must be grouped in a specific way for determining the function $F$ of the K-Map. Tables 2 and 3 show how both the K-map and truth graph, respectively, represent $000+001+110$. Figure 7 presents the result of combining the two truth paths in the first row of Table 3. The red and green paths share the same values, except for the one variable $\mathrm{C}$, which is eliminated. Figure 8 shows the results of combining the two paths. Then, we add the third path from the second row of Table 3 into the results of the combination of the two previous paths. Figure 9 illustrates the results of this combination that includes no common value for the black and blue paths to share. Therefore, this represents the simplest expression of $00+110(\overline{A B}+A B \bar{C})$.

Table 2. K-Map with truth values.

$$
\begin{aligned}
& \text { BC }
\end{aligned}
$$

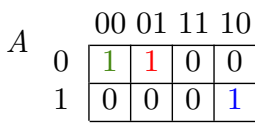

Table 3. K-Map with truth paths.

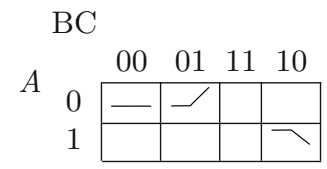




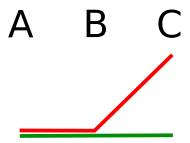

Fig. 7. Graph 1 and 2
A B C

Fig. 8. Results of Graph 1 and 2 (Color figure online)

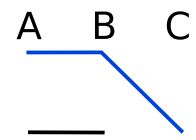

Fig. 9. Graph 3 and the results of Graph 1 and 2 (Color figure online)

\section{Truth Graph and K-Map (Four Variables)}

The same process is followed with four variables. Table 4 shows how the K-map represents all possible combinations, and Table 5 includes the same information represented by the truth graph.

Table 4. K-Map with values.

Table 5. K-Map with truth paths.

\begin{tabular}{cc|c|c|c|c|}
\multicolumn{2}{c}{$\mathrm{CD}$} & \multicolumn{2}{c}{} \\
\multicolumn{1}{c}{$A B$} & 00 & 01 & 11 & 10 \\
\cline { 3 - 6 } & 0000 & 0001 & 0011 & 0010 \\
\cline { 3 - 6 } & 01 & 0100 & 0101 & 0111 & 0110 \\
11 & 1100 & 1101 & 1111 & 1110 \\
10 & 1000 & 1001 & 1011 & 1010 \\
\cline { 3 - 5 } & & &
\end{tabular}

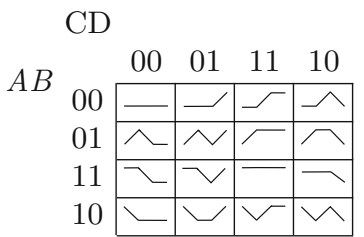

Let us assume that four combinations lead to true values, including $\overline{A B} C D+$ $\bar{A} B C D+A B C D+A \bar{B} C \bar{D}$. Figure 10 illustrates how the K-map and truth graph represent this information. Table 6 shows step-by-step how the combination of these values is minimized using our method. Step 1 combines the red and green paths so that we can eliminate variable B with the result shown in Step 2. Step 3 combines the result of step 1 with the blue path, and the value of variable $\mathrm{A}$ is eliminated from the blue path only because it passes through more variables (ABCD) compared to the black path (ACD). Step 4 shows the results of step 3 , and step 5 combines the result of step 3 with the yellow path. The yellow path shares only one variable with the other paths, which means no further minimization may occur. So, step 5 represents the final minimization of the truth graph in Fig. 10. As discussed above, we can select any two paths to begin the minimization process because any combination order leads to the same results. Moreover, the method is not affected by increasing the number of variables, which is a drawback of other methods. 

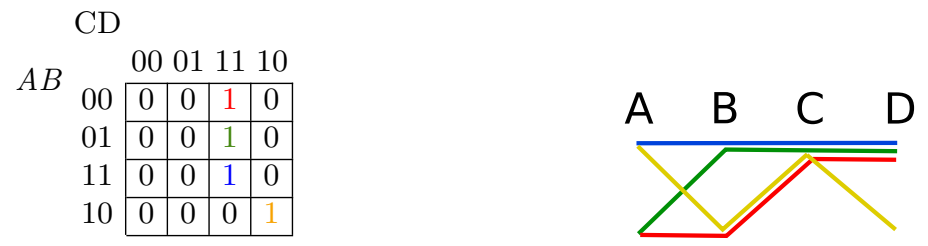

Fig. 10. Truth graph and K-map (4 variables).

Table 6. An example of minimzation process.

\begin{tabular}{|c|c|c|}
\hline Step & Symbolic Representation & Graphical Representation \\
\hline 1 & $\overline{A B} C D+\bar{A} B C D$ & $A \quad B \quad C \quad D$ \\
\hline 2 & $\bar{A} C D$ & $A \quad B \quad C \quad D$ \\
\hline 3 & $\bar{A} C D+A B C D$ & $A \quad B \quad C \quad D$ \\
\hline 4 & $\bar{A} C D+B C D$ & $A \quad B \quad C \quad D$ \\
\hline 5 & $\bar{A} C D+B C D+A \bar{B} C \bar{D}$ & A $\quad B \quad C \quad D$ \\
\hline
\end{tabular}

\section{Truth Graph (Six Variables)}

The truth graph method can be effectively applied to Boolean expressions with many variables. Figure 11 shows a truth graph representing an $A B C \overline{D E} F+$ $A \bar{B} C \overline{D E} F+\overline{A B C} D F$ expression. The blue and yellow paths pass through the same variables and values, except for variable B. Therefore, B is eliminated from the two paths, as shown in Fig. 12. The black path represents the result of the combination of the blue and yellow paths. The black dotted line indicates that variable $B$ is not included in the path. The red path passes through more than one value from the blue and yellow paths. Therefore, this represents the minimum expression as $A C \overline{D E} F+\overline{A B C} D E F$. 


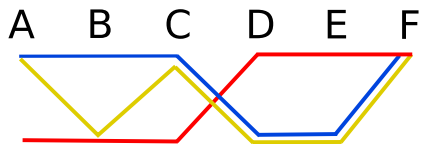

Fig. 11. Truth graph (6 variables).

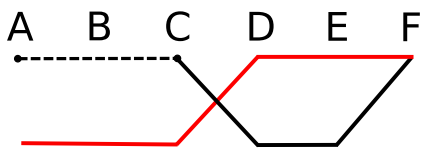

Fig. 12. Truth graph minimization result.

\section{Conclusion}

We introduced a novel method for minimizing Boolean expressions with graphs by considering the advantages of visual representations, including their simplicity, to overcome the limitations of truth tables and K-maps. Our next step will perform student tests to determine if an advantage exists for using these graph when minimizing Boolean algebra expressions compared to other representations, such as the K-map. Additional future work will be the implementation of tool support, as developing a tool that can automatically minimize drawn sketches will be valuable.

\section{References}

1. Ainsworth, S.: Deft: a conceptual framework for considering learning with multiple representations. Learn. Instr. 16(3), 183-198 (2006)

2. Ainsworth, S., Th Loizou, A.: The effects of self-explaining when learning with text or diagrams. Cogn. Sci. 27(4), 669-681 (2003)

3. Alharbi, E., Howse, J., Stapleton, G., Hamie, A., Touloumis, A.: Visual logics help people: an evaluation of diagrammatic, textual and symbolic notations. In: 2017 IEEE Symposium on Visual Languages and Human-Centric Computing (VL/HCC), pp. 255-259. IEEE (2017)

4. Alsallakh, B., Micallef, L., Aigner, W., Hauser, H., Miksch, S., Rodgers, P.: Visualizing sets and set-typed data: State-of-the-art and future challenges (2014)

5. Butcher, K.R.: Learning from text with diagrams: Promoting mental model development and inference generation. J. Educ. Psychol. 98(1), 182 (2006)

6. Chapman, P., Stapleton, G., Rodgers, P., Micallef, L., Blake, A.: Visualizing sets: an empirical comparison of diagram types. In: Dwyer, T., Purchase, H., Delaney, A. (eds.) Diagrams 2014. LNCS (LNAI), vol. 8578, pp. 146-160. Springer, Heidelberg (2014). https://doi.org/10.1007/978-3-662-44043-8_18

7. Cheng, P.C.-H.: Why diagrams are (sometimes) six times easier than words: benefits beyond locational indexing. In: Blackwell, A.F., Marriott, K., Shimojima, A. (eds.) Diagrams 2004. LNCS (LNAI), vol. 2980, pp. 242-254. Springer, Heidelberg (2004). https://doi.org/10.1007/978-3-540-25931-2_25

8. Cromley, J.G., Snyder-Hogan, L.E., Luciw-Dubas, U.A.: Cognitive activities in complex science text and diagrams. Contemp. Educ. Psychol. 35(1), 59-74 (2010)

9. Herman, G.L., Loui, M.C., Zilles, C.: Students' misconceptions about medium-scale integrated circuits. IEEE Trans. Educ. 54(4), 637-645 (2011)

10. Larkin, J.H., Simon, H.A.: Why a diagram is (sometimes) worth ten thousand words. Cogn. Sci. 11(1), 65-100 (1987)

11. Shimojima, A.: Semantic properties of diagrams and their cognitive potentials. In: Shimojima, A. (ed.) Center for the Study of Language and Information. CSLI Publications, California (2015) 
12. Stapleton, G., Jamnik, M., Shimojima, A.: Effective representation of information: generalizing free rides. In: Jamnik, M., Uesaka, Y., Elzer Schwartz, S. (eds.) Diagrams 2016. LNCS (LNAI), vol. 9781, pp. 296-299. Springer, Cham (2016). https://doi.org/10.1007/978-3-319-42333-3_28

13. Trotskovsky, E., Waks, S., Sabag, N., Hazzan, O.: Students' misunderstandings and misconceptions in engineering thinking. Int. J. Eng. Educ. 29(1), 107-118 (2013)

14. Veitch, E.W.: A chart method for simplifying truth functions. In: Proceedings of the 1952 ACM national meeting (Pittsburgh), pp. 127-133 (1952)

15. Zilles, C., Longino, J., Loui, M.: Student misconcfptions in an introductory digital logic design course. In: ASEE Annual Conference and Exposition, Conference Proceedings (2006)

Open Access This chapter is licensed under the terms of the Creative Commons Attribution 4.0 International License (http://creativecommons.org/licenses/by/4.0/), which permits use, sharing, adaptation, distribution and reproduction in any medium or format, as long as you give appropriate credit to the original author(s) and the source, provide a link to the Creative Commons license and indicate if changes were made.

The images or other third party material in this chapter are included in the chapter's Creative Commons license, unless indicated otherwise in a credit line to the material. If material is not included in the chapter's Creative Commons license and your intended use is not permitted by statutory regulation or exceeds the permitted use, you will need to obtain permission directly from the copyright holder.

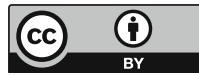

